\title{
The benefit of radiosurgery for ARUBA-eligible arteriovenous malformations: a practical analysis over an appropriate follow-up period
}

\author{
Daniel A. Tonetti, MD, ${ }^{1}$ Bradley A. Gross, MD, ${ }^{1}$ Kyle M. Atcheson, BS, ${ }^{1}$ Brian T. Jankowitz, MD, ${ }^{1}$ \\ Hideyuki Kano, MD, PhD, ${ }^{1}$ Edward A. Monaco III, MD, PhD, ${ }^{1}$ Ajay Niranjan, MD, ${ }^{1}$ \\ John C. Flickinger, MD, ${ }^{2}$ and L. Dade Lunsford, MD'
}

\begin{abstract}
The Center for Image-Guided Neurosurgery and Departments of ${ }^{1}$ Neurological Surgery and ${ }^{2}$ Radiation Oncology, University of Pittsburgh Medical Center, Pittsburgh, Pennsylvania
\end{abstract}

\begin{abstract}
OBJECTIVE The authors of this study found that, given the latency period required for arteriovenous malformation (AVM) obliteration after stereotactic radiosurgery (SRS), a study with limited follow-up cannot assess the benefit of SRS for unruptured AVMs.
METHODS The authors reviewed their institutional experience with "ARUBA (A Randomized Trial of Unruptured Brain Arteriovenous Malformations)-eligible" AVMs treated with SRS between 1987 and 2016, with the primary outcome defined as stroke (ischemic or hemorrhagic) or death (AVM related or AVM unrelated). Patients with at least 3 years of follow-up in addition to those who experienced stroke or died during the latency period were included. Secondary out- come measures included obliteration rates, patients with new seizure disorders, and those with new focal deficits without stroke.

RESULTS Of 233 patients included in this study, 32 had a stroke or died after SRS over the mean 8.4-year follow-up (14\%). Utilizing the $10 \%$ stroke or death rate at a mean 2.8-year follow-up for untreated AVMs in ARUBA, the rate in the authors' study is significantly lower than that anticipated at the 8.4 -year follow-up for an untreated cohort (14\% vs $30 \%$, $p=0.0003$ ). Notwithstanding obliteration, in this study, annualized rates of hemorrhage and stroke or death after 3 years following SRS were $0.4 \%$ and $0.8 \%$, respectively. The overall obliteration rate was $72 \%$; new seizure disorders, temporary new focal deficits without stroke, and permanent new focal deficits without stroke occurred in $2 \%$ of patients each. CONCLUSIONS After a sensible follow-up period exceeding the latency period, there is a lower rate of stroke/death for patients with treated, unruptured AVMs with SRS than for patients with untreated AVMs.

https://thejns.org/doi/abs/10.3171/2017.1.JNS162962

KEY WORDS arteriovenous malformation; AVM; ARUBA; SRS; stereotactic radiosurgery; hemorrhage; stroke; vascular disorders

$\mathrm{C}$ EREBRAL arteriovenous malformations (AVMs) are the most common arteriovenous shunts of the orrhagic event. It is well known that prior hemorrhage is a risk factor for subsequent bleeding. ${ }^{2,6,13}$ Despite the fact that AVMs are often diagnosed in a relatively young patient population, the management of unruptured AVMs has come under considerable scrutiny in the literature.

With the exception of a single analysis that reported a $0.9 \%$ annual hemorrhage rate for a selected cohort of un- ruptured AVMs,${ }^{13}$ the literature has been fairly consistent with a recent meta-analysis reporting an overall annual rupture risk of $2.2 \%$ for unruptured AVMs. ${ }^{4}$ This represents a single element of the natural history of AVMs that must be compounded with the prospective risk of headache development, seizures, psychological burden, and ischemic events that may occur once a patient is diagnosed with an intracerebral AVM.

Since stereotactic radiosurgery (SRS) requires a latency period to take effect, the benefit of this treatment modality 
cannot be realized for unruptured AVMs unless the patient is monitored for a reasonable period of time after the conclusion of the latency period. A Randomized Trial of Unruptured Brain Arteriovenous Malformations (ARUBA) compared any treatment modality for unruptured AVMs to no treatment, demonstrating a significantly lower rate of stroke or death in patients who did not undergo treatment. ${ }^{8}$ Unfortunately, with a mean follow-up period of less than 3 years, ARUBA would not be expected to demonstrate a benefit for SRS. The results from that study only serve to emphasize the obvious point that SRS may not be beneficial prior to the end of the latency period. Since patients have been counseled about this fact for decades, the study does not add to our knowledge about SRS and cannot be used to counsel patients about SRS since the follow-up period is inadequate.

In this report, we evaluated patients treated with SRS with at least 3 years of clinical follow-up. A recent multicenter study reported a $75 \%$ obliteration rate for ARUBAeligible AVMs treated with SRS. ${ }^{3}$ As such, the majority of patients in our analyzed cohort ought to have derived an angiographic benefit from treatment and would thus serve as a more reasonable comparison with the natural history of unruptured AVM. To provide a better parallel to the ARUBA study, specific parameters evaluated in that study, namely, stroke, death, and focal deficits without stroke, are evaluated and compared.

\section{Methods}

We reviewed our institution's prospectively collected Gamma Knife database for "ARUBA-eligible" AVMs treated initially with SRS from 1987 through October 2016. This specifically incorporated patients who were at least 18 years of age and harbored Spetzler-Martin Grade I-IV unruptured AVMs. ${ }^{12}$ Patients with at least 3 years of clinical follow-up in addition to any patient having an adverse event during the latency period (stroke, death, or SRS complication) were included in the analysis.

Background information, including patient age, sex, presentation modality (seizure, headache, focal deficit, asymptomatic, or other), AVM Spetzler-Martin grade, AVM size (stratified as less than or greater than $3 \mathrm{~cm}$ ), AVM location (supratentorial vs infratentorial; eloquent vs noneloquent), associated aneurysms, and venous drainage pattern (superficial only vs deep), were noted. Clinical and radiographic follow-up was also noted. Over this period, the primary outcome measure evaluated was stroke (ischemic/hemorrhagic) or death (AVM related or AVM unrelated). Rates of AVM obliteration, new seizures, and new focal deficits that occurred without stroke were also tallied as secondary outcome measures.

Given the lack of long-term natural history data that specifically provides stroke or death rates for unruptured AVMs, the ARUBA natural history cohort was used as a putative control. The rate of stroke and stroke or death in the study $(8 \%$ and $10 \%$ over a mean follow-up of 2.8 years, respectively) was annualized and then extrapolated along a linear, constant rate over an extended follow-up period for comparison with time points reached by patients in our cohort. Comparisons between outcome event rates be- tween the 2 cohorts were performed using a 2 -sided Fisher exact test and with relative risk ratios, with $\mathrm{p}<0.05$ defined as statistically significant.

\section{Results \\ Background and Demographics}

A total of 233 patients who were at least 18 years old and harbored Spetzler Grade I-IV unruptured AVMs that were treated initially with SRS were included in the study; 218 patients had at least 3 years of clinical follow-up after treatment, and 15 additional patients treated with SRS experienced a stroke or died prior to the 3-year followup. The mean patient age was 41.7 years and $55 \%$ of patients were female (Table 1). Most patients presented with seizure $(42 \%)$ or headache $(41 \%)$. The remaining patients were asymptomatic (10\%), had focal deficits $(10 \%)$, or had other symptoms (2\%). The majority of AVMs were small $(<3 \mathrm{~cm}, 58 \%) ; 73 \%$ were in eloquent locations, and $7 \%$ were infratentorial. Most AVMs had only superficial drainage (63\%), whereas $37 \%$ had deep venous drainage. According to the Spetzler-Martin grading system, most AVMs were Grade III (47\%) or Grade II (39\%). Eight percent of AVMs were Grade IV while only $5 \%$ were Grade I. Nine percent of AVMs had feeding artery or intranidal aneurysms. Thirty-two patients underwent repeat SRS (14\%), and 1 patient underwent a craniotomy for clot evacuation and excision of a subsequently hemorrhagic AVM.

\section{Primary Outcome Results}

Over a mean clinical follow-up period of 8.4 years (median 6.6 years), 32 of 233 patients either had a stroke or died (14\%). Specifically, 26 patients had a hemorrhagic stroke (11\%); 16 patients died as a result (7\%). One patient experienced an ischemic stroke. Five patients died of causes unrelated to their AVM (2\%), while 1 patient who had experienced a nonfatal hemorrhage subsequently died of a cause unrelated to the AVM. Of the 32 patients who underwent re-treatment with SRS, 3 patients $(9 \%)$ had a stroke or died. Considering the 218 patients with at least 3 years of follow-up after SRS, the annual hemorrhage rate after 3 years was $0.4 \%$; the annual stroke or death rate was $0.8 \%$.

\section{Factors Influencing the Primary Outcome}

Table 2 compares rates of the primary outcome between potentially relevant patient subgroups. AVM location, patient sex, and AVM size did not have a significant impact on the rate of stroke or death after SRS. Patients with AVMs with exclusively superficial drainage demonstrated a trend toward a lower rate of stroke or death $(10 \%$ vs $20 \%, p=0.05)$. Patients younger than 40 years had a significantly lower overall rate of stroke or death after treatment (7\% vs $20 \%$; RR $0.37,95 \%$ CI $0.17-0.80$; $\mathrm{p}=0.01$ ).

\section{Secondary Outcome Results}

The overall obliteration rate as determined by MRI and/or digital subtraction angiography was $72 \%$. Five patients $(2 \%)$ developed new seizures after treatment, $5(2 \%)$ 
TABLE 1. Baseline characteristics of patients in the current study with comparison to the no-treatment arm of patients in the ARUBA study

\begin{tabular}{lccc}
\hline \multicolumn{1}{c}{ Characteristic } & $\begin{array}{c}\text { Current } \\
\text { Study } \\
(\mathrm{n}=233)\end{array}$ & $\begin{array}{c}\text { ARUBA, } \\
\text { No-Treatment } \\
\text { Arm }(\mathrm{n}=109)\end{array}$ & $\mathrm{p}$ Value \\
\hline Mean age in yrs & 42 & 44 & \\
\hline Female sex & $129(55)$ & $44(40)$ & 0.01 \\
\hline Symptom & & & \\
$\quad$ Seizure & $99(42)$ & $45(41)$ & 0.91 \\
Headache & $96(41)$ & $59(54)$ & 0.03 \\
Focal deficit & $23(10)$ & $10(9)$ & 1.0 \\
$\quad$ Other & $5(2)$ & $8(7)$ & 0.03 \\
\hline SM grade & & & \\
I & $12(5)$ & $33(30)$ & $<0.0001$ \\
II & $92(39)$ & $27(25)$ & 0.008 \\
III & $109(47)$ & $34(31)$ & 0.007 \\
IV & $19(8)$ & $15(14)$ & 0.12 \\
\hline AVM size <3 cm & $134(58)$ & $60(55)$ & 0.73 \\
Left-sided AVM & $125(54)$ & $50(46)$ & 0.20 \\
Infratentorial location & $17(7)$ & $5(5)$ & 0.48 \\
Eloquent location & $169(73)$ & $51(47)$ & $<0.0001$ \\
\hline Associated aneurysm & $22(9)$ & $21(19)$ & 0.01 \\
\hline Superficial-only drainage & $146(63)$ & $69(63)$ & 1.0 \\
Any deep drainage & $87(37)$ & $40(37)$ & 1.0 \\
\hline
\end{tabular}

$\mathrm{SM}=$ Spetzler-Martin.

Values are presented as the number of patients (\%) unless stated otherwise.

developed new permanent focal deficits without stroke, and 4 patients (2\%) developed new temporary focal deficits without stroke.

\section{Comparison With the ARUBA No-Treatment/Natural History Cohort}

Patients in the current study were of a similar age to those in the ARUBA (Table 1). However, a greater proportion of patients in our study were female, and a smaller proportion had headaches. Importantly, in our study, there was a significantly smaller proportion of Spetzler Grade I AVMs and a significantly greater proportion of Spetzler Grade II or III AVMs compared with the ARUBA. In addition, there was specifically a greater proportion of eloquent AVMs in our study, whereas there was no significant difference in the proportion of AVMs with superficial venous drainage or nidus size less than $3 \mathrm{~cm}$. There was a significantly lower proportion of AVMs with associated aneurysms in our study.

The $14 \%$ stroke or death rate in our study with a mean follow-up period of 8.44 years does not significantly differ from the $10 \%$ stroke or death rate for the no-treatment arm in ARUBA after a follow-up period of only 2.80 years (RR 1.36, 95\% CI 0.71-2.60; $\mathrm{p}=0.35$ ). Assuming an annualized, constant stroke or death rate for the untreated cohort, one would anticipate a 30\% stroke or death rate for this group after a mean follow-up of 8.44 years. For this direct, putative comparison, the rate of stroke or death in this study is significantly lower (14\% vs $30 \%$; RR 0.45 , 95\% CI 0.30-0.70; $\mathrm{p}=0.0003$ ).
TABLE 2. Subgroup analysis of stroke or death rates after SRS

\begin{tabular}{lccc}
\hline \multicolumn{1}{c}{ Variable } & $\begin{array}{c}\text { Stroke/ } \\
\text { Death }(\%)\end{array}$ & RR $(95 \%$ Cl) & p Value \\
\hline Age $<40$ yrs & $8 / 110(7)$ & $0.37(0.17-0.80)$ & 0.01 \\
Age $\geq 40$ yrs & $24 / 123(20)$ & & \\
\hline Female & $15 / 129(12)$ & $0.71(0.37-1.36)$ & 0.30 \\
Male & $17 / 104(16)$ & & \\
\hline SM Grade I or II & $10 / 104(10)$ & $0.56(0.28-1.14)$ & 0.11 \\
SM Grade III or IV & $22 / 129(17)$ & & \\
\hline Supratentorial & $29 / 216(13)$ & $0.76(0.26-2.24)$ & 0.62 \\
Infratentorial & $3 / 17(18)$ & & \\
\hline Size $<3$ cm & $15 / 134(11)$ & $0.65(0.34-1.24)$ & 0.19 \\
Size $>3$ cm & $17 / 99(17)$ & & \\
\hline Eloquent & $19 / 169(11)$ & $0.55(0.29-1.05)$ & 0.07 \\
Noneloquent & $13 / 64(20)$ & & \\
\hline Superficial-only drainage & $15 / 146(10)$ & $0.53(0.28-1.0)$ & 0.05 \\
Any deep drainage & $17 / 87(20)$ & & \\
\hline
\end{tabular}

\section{Discussion}

While the practitioner's goal in the management of an AVM is angiographic obliteration without complications, a more practical clinically oriented goal is the prospective avoidance of neurological sequelae, most commonly in the form of stroke or even death. Multiple radiosurgical publications have evaluated obliteration rates for unruptured AVMs, including a recent multicenter registry of ARUBA-eligible unruptured AVMs, which included a significant number of patients from our institution. ${ }^{3}$ In that study, radiosurgery achieved a $75 \%$ obliteration rate over a mean follow-up period of 86 months for patients who met ARUBA criteria. The authors hypothesized that, because $30 \%$ of the patients in this study showed unfavorable outcome (defined as incomplete obliteration, hemorrhagic stroke, or a treatment-related complication), it would take 15-20 years of follow-up to establish a benefit of SRS over medical management; ${ }^{3}$ in contrast, this analysis demonstrates a benefit and at an earlier follow-up than anticipated. Other studies not specifically looking at radiosurgical cohorts have used the modified Rankin Scale or persistent neurological deficits as the primary outcome., ${ }^{911}$ However, only a paucity of studies have evaluated radiosurgical outcomes for unruptured AVMs in terms of ARUBA outcome parameters; the one study meeting these criteria retrospectively reviewed 174 ARUBA-eligible patients and demonstrated an annual hemorrhagic stroke risk of $2 \%$ for the first 5 years after SRS, declining to $0.2 \%$ per year from Years 6 to 10 post-SRS. ${ }^{10}$

The aim of the ARUBA study was to compare the risk of no treatment for unruptured AVMs to prophylactic intervention. Early analysis of ARUBA suggested that patients with unruptured AVMs who underwent intervention suffered the primary outcome, death or symptomatic stroke, at a greater than 3 -fold higher rate than those patients who were managed without intervention (HR 3.70, 95\% CI 1.85-7.14). ${ }^{8}$ Criticisms of the ARUBA study have been well documented; $1,5,7,14$ chiefly, the short mean followup of 33.3 months (2.8 years) biases the results toward capturing early adverse clinical events, including stroke 
or death in those patients randomized to the intervention arm, where the risk is expected to be highest in the early posttreatment period for patients undergoing endovascular management or microsurgical removal.

In this study, we present an analysis of ARUBA-eligible patients who underwent SRS with long-term follow-up and compare the rates of symptomatic stroke or death in our radiosurgical population, the primary ARUBA outcome, to the no-treatment arm of the ARUBA trial. Rates of stroke or death are similar over the first 3 years-approximately the length of the ARUBA trial follow-up period and approximately the length of the latency period-but significantly diverge as follow-up lengthens (Fig. 1). The annual hemorrhage rate after 3 years post-SRS was $0.4 \%$, whereas the annual stroke or death rate was $0.8 \%$ after 3 years in our population. These outcomes are similar to those of other studies that have evaluated outcomes after SRS. ${ }^{10}$ It is interesting to note that patients in our study, compared with those in the ARUBA, harbored more surgically challenging AVMs as defined by the Spetzler-Martin grade.

Treatment of unruptured AVMs is predicated on reducing the risk of stroke or death for the remainder of these patients' lives. Given the latency period after SRS prior to obliteration, patients are expected to derive more benefit from this interventional approach when these patients undergo long-term follow-up. Our data support this hypothesis, as subgroup analysis demonstrates that patients who are young $(<40$ years) are significantly less likely to experience a stroke or die after radiosurgery than those who are 40 years or older $(p=0.01)$, likely due to the fact that patients in our study who died of causes unrelated to the AVM were preferentially older. Our study suggests that SRS has even more practicality when the patient is young. Furthermore, patients with exclusively superficial venous drainage $(\mathrm{p}=0.05)$ demonstrated a trend toward a lower rate of stroke or death, which is in concordance with prior literature.

In an effort to simulate long-term follow-up for a natural history cohort, we used linear extrapolation of the rate of stroke or death from the no-intervention arm of the ARUBA trial. There are several potential sources of error with this assumption. It is important to emphasize that we in fact excluded patients with less than 3 years of follow-up who did not experience a stroke or die, somewhat inflating our reported rate of stroke or death for the overall treated cohort at our institution. However, our assumption of a constant annual hemorrhage rate for untreated AVMs may in fact be conservative, given the potential for the development/formation of high-risk features, such as aneurysms or venous outflow obstruction. In addition, the cumulative risk of death may also increase with time as patients age. As with all assumptions, the validity of the assumption of a linear rate of stroke or death over lengthy time periods is not yet known, and we await long-term results from the ARUBA cohort to answer this question.

\section{Study Limitations}

This study is limited by its retrospective nature, which subjects our study to obvious selection bias and treatment preferences of the physicians involved in the care of these

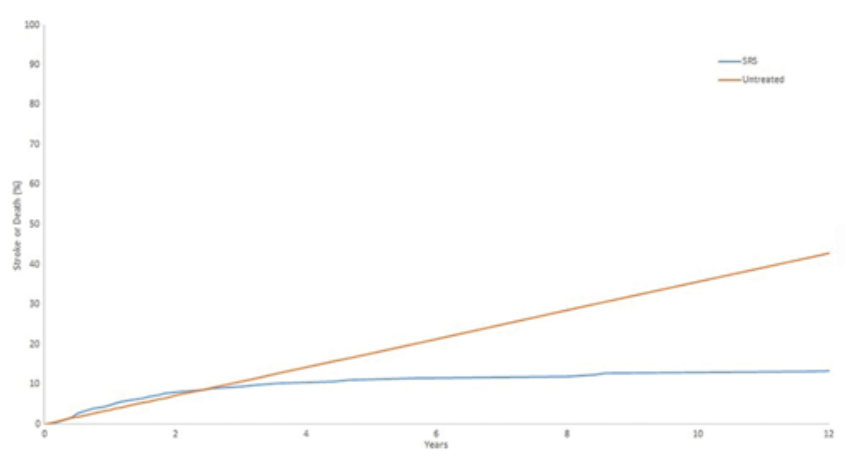

FIG. 1. Cumulative rate of stroke or death for ARUBA-eligible patients who underwent SRS compared with the no-treatment arm of ARUBA, demonstrating divergence of the rate of stroke or death as follow-up lengthens. Figure is available in color online only.

patients. Accordingly, there are differences in the AVMs of the patient populations; our population experienced significantly fewer AVM-associated aneurysms than the ARUBA cohort, which may reflect the desire to treat those AVMs with interventions other than radiosurgery. While this is a retrospective review, it is a review of patients who were prospectively entered into a database registry over the past 25 years and regularly reassessed to perform ongoing updates on the results of AVM radiosurgery at our center. This assessment provides a longer postintervention follow-up period than any other published data related to the ARUBA study using the same outcome established by ARUBA as a comparison. However, this is a single-institution study, and our institution has considerable experience with SRS for AVMs. This may bias the results of our study toward better outcomes for our radiosurgical cohort and may limit the generalizability of our study.

\section{Conclusions}

In our single-institution experience, radiosurgery decreases the rate of hemorrhage/stroke and death from unruptured AVMs over a sensible, long-term follow-up period compared with a putative, no-treatment control group.

\section{References}

1. Cockroft KM, Jayaraman MV, Amin-Hanjani S, Derdeyn $\mathrm{CP}$, McDougall CG, Wilson JA: A perfect storm: how a randomized trial of unruptured brain arteriovenous malformations' (ARUBA's) trial design challenges notions of external validity. Stroke 43:1979-1981, 2012

2. da Costa L, Wallace MC, Ter Brugge KG, O'Kelly C, Willinsky RA, Tymianski M: The natural history and predictive features of hemorrhage from brain arteriovenous malformations. Stroke 40:100-105, 2009

3. Ding D, Starke RM, Kano H, Mathieu D, Huang P, Kondziolka D, et al: Radiosurgery for cerebral arteriovenous malformations in A Randomized Trial of Unruptured Brain Arteriovenous Malformations (ARUBA)-eligible patients: a multicenter study. Stroke 47:342-349, 2016

4. Gross BA, Du R: Natural history of cerebral arteriovenous malformations: a meta-analysis. J Neurosurg 118:437-443, 2013

5. Gross BA, Scott RM, Smith ER: Management of brain arteriovenous malformations. Lancet 383:1635, 2014

6. Kim H, Sidney S, McCulloch CE, Poon KY, Singh V, John- 
ston SC, et al: Racial/ethnic differences in longitudinal risk of intracranial hemorrhage in brain arteriovenous malformation patients. Stroke 38:2430-2437, 2007

7. Mathiesen T: Arguments against the proposed randomised trial (ARUBA). Neuroradiology 50:469-471, 2008

8. Mohr JP, Parides MK, Stapf C, Moquete E, Moy CS, Overbey JR, et al: Medical management with or without interventional therapy for unruptured brain arteriovenous malformations (ARUBA): a multicentre, non-blinded, randomised trial. Lancet 383:614-621, 2014

9. Nerva JD, Mantovani A, Barber J, Kim LJ, Rockhill JK, Hallam DK, et al: Treatment outcomes of unruptured arteriovenous malformations with a subgroup analysis of ARUBA (A Randomized Trial of Unruptured Brain Arteriovenous Malformations)-eligible patients. Neurosurgery 76:563-570, 2015

10. Pollock BE, Link MJ, Brown RD: The risk of stroke or clinical impairment after stereotactic radiosurgery for ARUBAeligible patients. Stroke 44:437-441, 2013

11. Rutledge WC, Abla AA, Nelson J, Halbach VV, Kim H, Lawton MT: Treatment and outcomes of ARUBA-eligible patients with unruptured brain arteriovenous malformations at a single institution. Neurosurg Focus 37(3):E8, 2014

12. Spetzler RF, Martin NA: A proposed grading system for arteriovenous malformations. J Neurosurg 65:476-483, 1986

13. Stapf C, Mast H, Sciacca RR, Choi JH, Khaw AV, Connolly ES, et al: Predictors of hemorrhage in patients with untreated brain arteriovenous malformation. Neurology 66:1350-1355, 2006
14. Starke RM, Sheehan JP, Ding D, Liu KC, Kondziolka D, Crowley RW, et al: Conservative management or intervention for unruptured brain arteriovenous malformations. World Neurosurg 82:e668-e669, 2014

\section{Disclosures}

Dr. Lunsford reports the following: consultant for Best Doctors, DSMB, and Advance Medical; and consultant for, stockholder in, and member of the Data Safety Monitoring Board of AB Elekta.

\section{Author Contributions}

Conception and design: Tonetti, Gross, Niranjan, Flickinger, Lunsford. Acquisition of data: Tonetti, Atcheson, Monaco. Analysis and interpretation of data: Tonetti, Gross, Lunsford. Drafting the article: Tonetti, Gross, Atcheson, Niranjan. Critically revising the article: all authors. Reviewed submitted version of manuscript: Tonetti, Gross, Atcheson, Jankowitz, Kano, Monaco, Niranjan, Flickinger. Approved the final version of the manuscript on behalf of all authors: Tonetti. Statistical analysis: Tonetti, Gross. Administrative/technical/material support: Tonetti, Gross, Lunsford. Study supervision: Tonetti, Gross, Niranjan, Flickinger, Lunsford.

\section{Correspondence}

Daniel Tonetti, Department of Neurological Surgery, University of Pittsburgh, Ste. B-400, UPMC Presbyterian, 200 Lothrop St., Pittsburgh, PA 15213. email: tonettida@upmc.edu. 